\title{
Microbiological profile and changing trends of antibiogram profile in patients of chronic suppurative otitis media in a tertiary hospital in NCR region
}

\author{
Ritu Gupta ${ }^{1}$, Varun Goel ${ }^{2, *}$, O. N. Sinha ${ }^{3}$, Dakshina Bisht ${ }^{4}$ \\ ${ }^{1}$ Post Graduate Student, ${ }^{2}$ Assistant Professor, ${ }^{4}$ Professor and Head, Dept. of Microbiology, Santosh Medical College \& Hospital, \\ Ghaziabad Uttar Pradesh, ${ }^{3}$ Professor and Head, Dept. of Otorhinolaryngology (ENT), Santosh Medical College \& Hospital,
} Ghaziabad, Uttar Pradesh, India

*Corresponding Author:

Email: drvarun21@gmail.com

\begin{abstract}
Introduction: Otitis media $(\mathrm{OM})$ is a major health issue involving mainly middle ear and is quite prevalent in developing countries in the low socioeconomic group. The aim of this study was to isolate the organisms associated with chronic suppurative otitis media (CSOM) and to detect the antibiogram of the aerobic isolates.

Methods: A total of 150 patients clinically diagnosed with CSOM were enrolled in the study and the samples were obtained from each patient using sterile cotton swabs and cultured for microbial flora. Drug susceptibility testing for aerobic isolates was conducted using Kirby-Bauer disc diffusion method.

Results: Out of the $119(79.33 \%)$ were culture positive, of which $93(78.15 \%)$ were pure bacterial isolates and $26(27.95 \%)$ were mixed cultures. Bacterial isolates in pure cultures were Staphylococcus aureus in 46 isolates (49.46\%), Pseudomonas aeruginosa $22(23.6 \%)$. Antimicrobial profile of aerobic isolates revealed maximum sensitivity to amikacin (95.5\%), ceftriaxone (83.4\%) and gentamicin $(82.7 \%)$.

Conclusions: Knowing the etiological agents of CSOM and their antimicrobial susceptibility is of essential importance for an effective treatment, prevention of both complications and development of antibiotic resistance and finally, the reduction of the treatment costs.
\end{abstract}

Keywords: Attico-antral, Chronic suppurative otitis media, Pseudomonas aeruginosa, Staphylococcus aureus.

\section{Introduction}

Chronic suppurative otitis media is defined as a perforation of the tympanic membrane with persistent drainage of pus from the middle ear lasting from 6 to 12 weeks. It is a major cause of acquired hearing impairment of varying severity mostly in developing countries. ${ }^{1,2}$ Hearing loss in children is likely to affect the language and cognitive development. WHO has classified the countries into different groups according to the prevalence of chronic suppurative otitis media (CSOM) like Highest (> 4\%), High (2-4\%), Low (1$2 \%$ ), and Lowest $(<1 \%)$, in which India comes under Highest category (WHO, 1998). Prevalence of CSOM in India according to the $\mathrm{WHO}$ reports is $7.8 \%$ which puts India amongst the group with the highest prevalence and hence demands urgent attention to deal with a massive public health problem. ${ }^{3}$

The incidence of this disease is higher in developing world because of malnutrition, overcrowding, poor hygiene, inadequate health care, and recurrent upper respiratory tract infection. ${ }^{4}$ The urban to rural ratio of the disease is $1: 2$ and the poorer rural communities have the highest prevalence. ${ }^{5}$

The objective of this study was to investigate the clinico-microbiological profile of CSOM and to analyze the susceptibility pattern of the aerobic bacterial isolates at our tertiary care centre, so that an antibiotic policy is formulated for CSOM, for better patient management.

\section{Methods}

This cross sectional study was conducted for a period of 14 months from March 2016 to April 2017 at a tertiary care teaching hospital. Ethical clearance was obtained from the Institutional Ethical Committee. The study group included 150 randomly selected patients between 1 and 78 years, who were clinically diagnosed with CSOM. Inclusion criteria included patients presenting with tympanic perforation and ear discharge of more than 3 months. Only those patients who were not on any antibiotics (oral and systemic) in the previous five days were included in the study. Informed consent was obtained at enrollment.

Before collecting the aural discharge, the external auditory canal was cleared of cerumen, swabbed with boric acid spirit and allowed to dry. The middle ear discharge was then aseptically collected by the otorhino-laryngologist from the tympanic cavity with a thin, sterile cotton swab (Hi Media, Mumbai, India). Also, care was taken not to touch the tympanic membrane or the external auditory canal during sample collection. The specimens so collected were transported immediately to the microbiology laboratory for further processing.

The swabs were inoculated onto sheep blood agar, chocolate agar and Mac Conkey agar for aerobic culture and the inoculated plates were incubated at $37^{\circ} \mathrm{C}$ for 24-48 h with 5\% carbon dioxide enrichment for blood agar and chocolate agar plates. The aerobic bacterial isolates were identified according to standard 
bacteriological techniques. Antimicrobial susceptibility testing for aerobic bacterial isolates was done by KirbyBauer disc diffusion method following the Clinical Laboratory Standards Institute (CLSI) guidelines. ${ }^{6}$ Methicillin resistance among Staphylococcus aureus strains was detected by cefoxitin disc test. ESBL production was considered positive if zone diameter of inhibition around the disc of ceftazidime clavulanic acid and cefotaxime clavulanic acid $\geq 5 \mathrm{~mm}$ than the ceftazidime or cefotaxime disc alone. ${ }^{7}$ AmpC production was suggested when the isolate was resistant to cefoxitin (Zone diameter $\leq 14 \mathrm{~mm}$ ) but susceptible to cefepime (Zone diameter $\geq 25 \mathrm{~mm}$ ). Isolates showing reduced susceptibility to imipenem were selected for detection of MBL enzymes by imipenem-EDTA combined disk method. ${ }^{8}$

A standard bacteriological procedure was followed to keep the quality of all laboratory tests. American Type Culture Collection (ATCC) strains (E. coli ATCC 25922, $P$. aeruginosa ATCC 27853, S. aureus ATCC25923, K. pneumoniae ATCC 700603 and $P$. mirabilis ATCC 35659) were used as controls for culture and sensitivity testing. The data was analyzed by using Statistical Package for Social Sciences (SPSS) version 11 and the prevalence of organisms was determined and expressed in percentage.

\section{Results}

Out of the $119(79.33 \%)$ were culture positive, of which $93(78.15 \%)$ were pure bacterial isolates and 26 $(27.95 \%)$ were mixed cultures. In clinically diagnosed cases of CSOM, the age varied from 0 to 70 years. Maximum patients were in the age group of 21- 30 years $(46 \%)$ followed by age group 31-40 (24\%) years and those between 11- $20(12.66 \%)$ years. [Table1] Among the COSM patients, $122(81 \%)$ were from the rural area while $28(19 \%)$ belong to the urban area. Among the 150 cases, right ear was involved in 55 $(37 \%)$ cases while left ear was involved in $82(54 \%)$ cases with $13(9 \%)$ cases of bilateral involvement. Males (55\%) were more commonly affected than females $(45 \%)$ and the sex ratio male: female was 1.2:1. Bacterial isolates in pure cultures were Staphylococcus aureus in 46 isolates $(49.46 \%), P$. aeruginosa 22 (23.6\%), Klebsiella pneumonia (13.97\%), Proteus vulgaris 8 (8.6\%), Citrobacter freundii 2 (2.15\%), Acinetobacter baumanii $1(1.07 \%)$ and Enterococcus spp.1 (1.07\%). [Table 2] Among the mixed growth cultures predominate bacteria were Staphylococcus aureus $(42.3 \%)$ and P. aeruginosa (34.61\%).

S. aureus isolates were found to be $100 \%$ sensitive to Vancomycin followed by Clindamycin (92.6\%), ceftazidime $(89.7 \%)$, cefoxitin and amikacin whereas $11.5 \%$ of $S$. aureus isolates were MRSA strains which were found to be $100 \%$ sensitive to Vancomycin and $75 \%$ sensitive to Clindamycin. Pseudomonas aeruginosa shows maximum sensitivity to imipenem $(92.5 \%)$ followed by piperacillin-tazobactam (75\%), amikacin $(70 \%)$ and ceftazidime (70\%). Klebsiella pneumoniae showed $100 \%$ sensitivity to amikacin, imipenem and piperacillin tazobactam. E.coli was $100 \%$ sensitive to piperacillin tazobactam, ceftazidime, imipenem and cefoxitin whereas $66 \%$ sensitive to amikacin, Amoxiclav and Gentamycin imipenem and cefoxitin. Proteus vulgaris was $100 \%$ sensitive ceftazidime, imipenem and cefoxitin whereas $66 \%$ sensitive to amikacin, Amoxiclav and Gentamycin. Citrobacter freundii was $100 \%$ resistant to Ciprofloxacin whereas Acinetobacter baumanii was $100 \%$ resistant to Gentamycin. [Graph 1] Result of $\beta$ lactamase production is shown in Table 3.

Table 1: Socio demographic and clinical manifestation of patients with ear discharge

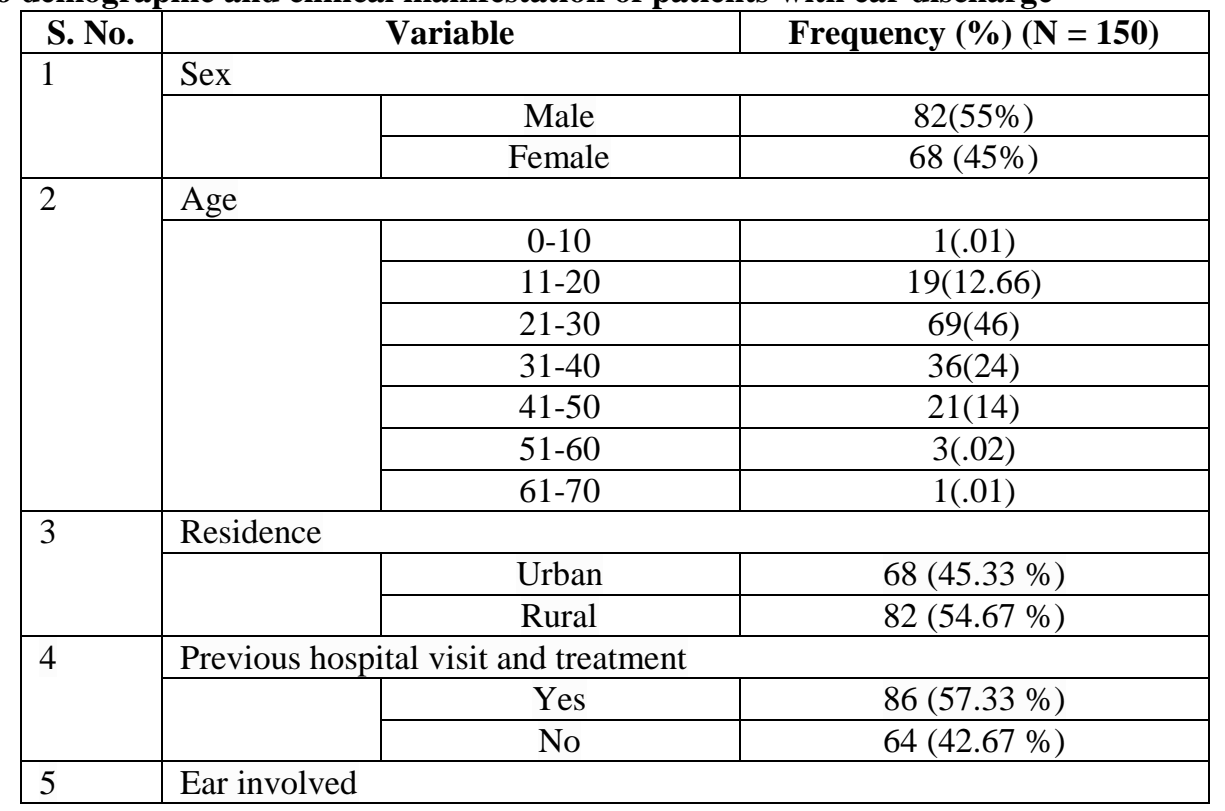




\begin{tabular}{|l|c|c|c|}
\hline \multirow{2}{*}{} & & Right & $55(37 \%)$ \\
\cline { 3 - 4 } & & Left & $82(54 \%)$ \\
\cline { 3 - 4 } & \multicolumn{2}{|c|}{ Both } & $13(9 \%)$ \\
\hline 6 & Reason to visit ENT clinic & $51(34 \%)$ \\
\cline { 2 - 3 } & & Self & $99(66 \%)$ \\
\cline { 2 - 3 } & & Referred & \\
\hline
\end{tabular}

Table 2: Shows different types of isolates from CSOM patients

\begin{tabular}{|l|c|c|}
\hline S. No. & Types of organisms & Number of isolates \\
\hline 1 & Aerobic bacteria & $119(79.33 \%)$ \\
\hline 2 & Staphylococcus aureus & $61(49.46 \%)$ \\
\hline 3 & P. aeruginosa & $34(23.6 \%)$ \\
\hline 4 & Klebsiella pneumonia & $17(13.97 \%)$ \\
\hline 5 & Proteus vulgaris & $10(8.6 \%)$ \\
\hline 6 & Citrobacter freundii & $2(2.51 \%)$ \\
\hline 7 & Acinetobacter baumanii & $1(1.07 \%)$ \\
\hline 8 & Enterococcus Spp. & $1(1.07 \%)$ \\
\hline
\end{tabular}

Table 3: Result of $\beta$-lactamase Production in Gram negative bacilli

\begin{tabular}{|l|c|c|}
\hline S. No. & $\boldsymbol{\beta}$-lactamase & Number of isolates (\%) \\
\hline 1 & ESBL combined disk test & $14(16.09)$ \\
\hline 2 & MBL (Imipenem-EDTA combined disk method) & $07(9.07)$ \\
\hline 3 & Amp C disk test & $02(2.09)$ \\
\hline
\end{tabular}

Graph 1: Antibiotic sensitivity pattern of Gram negative bacilli

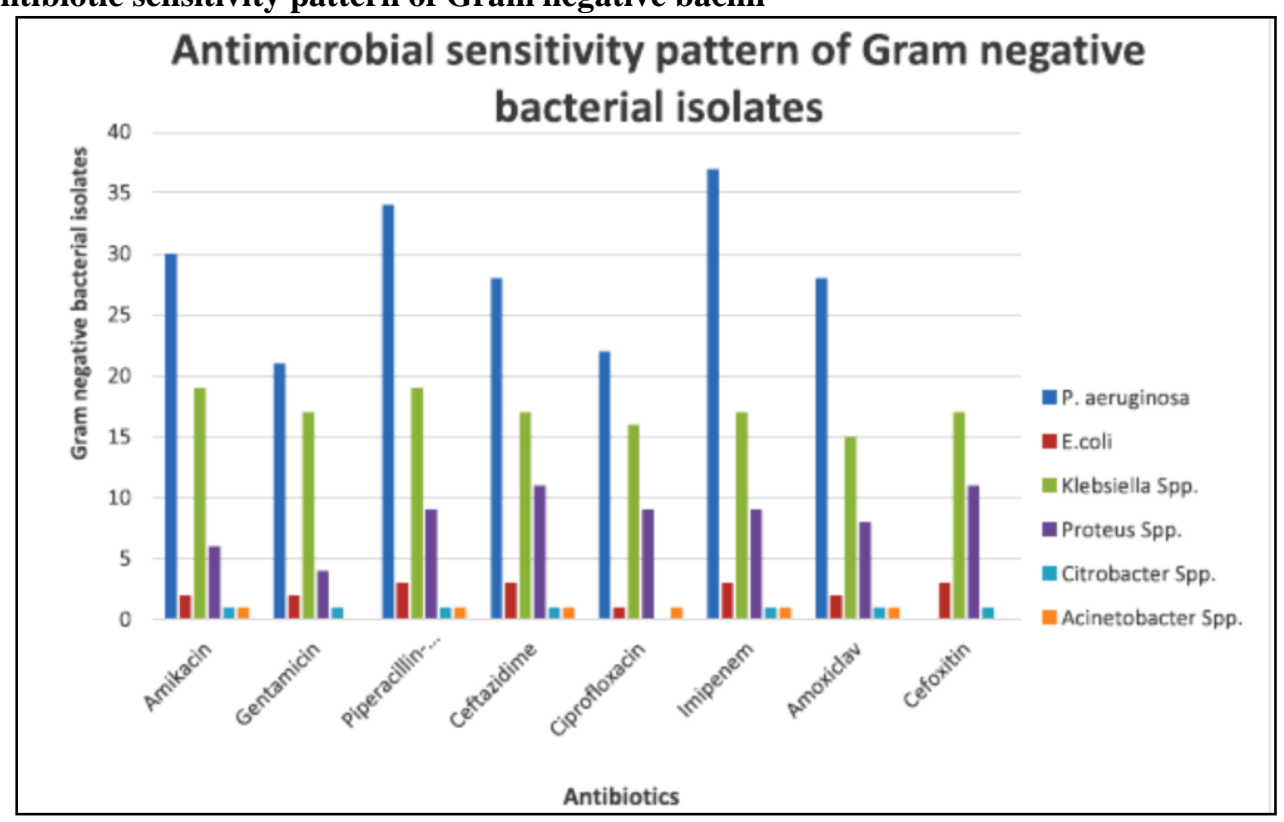

\section{Discussion}

CSOM is a major public-health problem, and India is one of the countries with high-prevalence where urgent attention is needed. ${ }^{9} \mathrm{CSOM}$ is an important cause of preventable hearing loss and of serious concern in pediatric age group because it may have long-term effects on early communication, language development, educational process, auditory processing and physiological and cognitive development. ${ }^{10}$ Early, microbiological diagnosis ensures prompt and effective treatment to avoid such complications. A total of 150 cases of CSOM were undertaken in the study and it was found that as compared to females, males were found to be more associated with CSOM in our study which is analogous to studies done on CSOM cases. ${ }^{9,11}$ whereas in contrast to our study, females were more affected than males in another study. ${ }^{12}$ On the other hand, another study reported no difference in the prevalence of otitis media among sexes. ${ }^{13}$ Our results may be due to outdoor working habits of males which is usually higher in poor developing areas as well as due to geographical variations. 
The highest percentage of patients in CSOM was observed in the age group of 21-30 years and 31-40 years as seen in other studies. ${ }^{13}$ While Loy et al, (2002) and Gupta $\mathrm{V}$ et al, (1998) report the highest percentage of presentation in the fourth decade. ${ }^{14,15}$

High rate of unilateral involvement of ear discharge (91\%) was observed along with low rate of bilateral involvement of ear discharge (9\%) among CSOM patients. In accordance with our study, high rate of unilateral involvement (82\%) was also recorded by Nwokoye et al and Akinpelu et al cases of Otitis media. ${ }^{16,17}$

In this study $79.33 \%$ CSOM cases were culture positive while no growth was observed in $20.15 \%$ of cases as a similar study by Kumar et a ${ }^{18}$ while in contrast to present study, Singhal et $\mathrm{al}^{19}$ showed $94.67 \%$ of culture positive and $5.33 \%$ of culturenegative cases.

The most common bacterial isolate in CSOM in our present study was $S$. aureus in $(49.46 \%)$ of which $11.5 \%$ were MRSA, followed by Pseudomonas aeruginosa (23.6\%) similar to few other studies. ${ }^{20,}{ }^{21}$ In contrast to our study, few studies have demonstrated Pseudomonas aeruginosa to be the most common isolate, followed by $S$. aureus. ${ }^{22}$ These studies depict that the microbial profile vary between different regions based on patient population and geographical distribution and hence necessitates the need for frequent analysis and update of the microbial profile in every region.

In present study, $S$. aureus isolates were found to be $100 \%$ sensitive to Vancomycin followed by Clindamycin (92.6\%), ceftazidime (89.7\%). 8 (11.5\%) isolates of $S$. aureus isolates were identified as MRSA strains and were found to be $(100 \%)$ sensitive to Vancomycin and $75 \%$ sensitive to Clindamycin. Similarly, Rakesh Kumar et $\mathrm{al}^{23}$ observed that $S$. aureus was100\% sensitive to Linezolid and Vancomycin followed by Cefoperazone / Sulbactam, amikacin, Clindamycin, piperacillin / tazobactam, Erythromycin and Ofloxacin. Pseudomonas aeruginosa shows maximum sensitivity to imipenem $(92.5 \%)$ followed by piperacillin-tazobactam (75\%), amikacin $(70 \%)$ and ceftazidime $(70 \%)$. In contrast to our study Pseudomonas aeruginosa showed $100 \%$ susceptibility to piperacillin-tazobactam and ceftazidime-Clavulanic acid, imipenem (96\%), Ciprofloxacin (89\%) and amikacin $(82 \%)$ in the study done by Jeya Kumari, D et al. $^{24}$

In our study, ESBL were detected in $14(18.42 \%)$ of 87 the Gram-negative bacilli, of which 12 ESBL were Pseudomonas aeruginosa and 2 isolates were Klebsiella spp. However, higher incidence of ESBL among Pseudomonas aeruginosa from $42 \%$ to $64 \%$ has been reported in other studies. ${ }^{7,25}$ In our study MBL were detected in 7 (9.21\%) Gram negative bacilli by imipenem-EDTA combined disc test of which $3(7.5 \%)$ were Pseudomonas aeruginosa, $2(10.5 \%)$ were
Klebsiella pneumoniae and $2(18.1 \%)$ were Proteus vulgaris. In contrast, MBL producers were not detected in a study done by Khatoon et al. ${ }^{26}$

In our study AmpC were detected in $1.33 \%$ Klebsiella pneumonia whereas $20 \%$ AmpC were detected in study done by Khatoon et al. ${ }^{26}$ The sensitivity pattern shows that it is increasingly becoming more resistant to the common and routine antibiotics used in the ENT OPD. One reason for this could be the fact that most of these patients usually present in the ENT OPD after the previous treatments have failed and another key factor is that the cultures are mostly requested when commonly used drugs have failed to eradicate past infection.

Continuous and periodic evaluation of microbiological profile and antimicrobial sensitivity pattern of bacterial is essential for optimum management of CSOM patients. In conclusion, it is necessary to highlight the antibiotic crisis as a result of the emergence of many genera of bacteria that are multi drug resistant. We believe that our data may contribute to an effective medical management of CSOM.

\section{References}

1. Mansoor T, Musani MA, Khalid G, Kamal M. Pseudomonas aeruginosa in chronic suppurative otitis media: Sensitivity spectrum against various antibiotics in Karachi. J Ayub Med Coll Abbottabad. 2009;21:120-3.

2. Iqbal K, Khan M, Satti L. Microbiology of Chronic Suppurative Otitis Media: Experience Dera Ismail Khan. Gomal J Med Sci 2011;9(2):189-93.

3. Sharma K, Oberoi L, Narula V. Present scenario of microbiological pattern in chronic suppurative otitis media and its management guidelines. J Acad Cli Microb 2017;19(1):47-53.

4. Qureishi A, Lee Y, Belfield K, Birchall JP, Daniel M. Update on otitis media prevention and treatment. Infect Drug Resist 2014;7:15-24.

5. Monasta L, Ronfani L, Marchetti F, Montico M, Brumatti LV, Bavca A et al; Burden of disease caused by otitis media: systematic review and global estimates. PLoS One 2012;7(4):e36226.

6. CLSI. CLSI Document M100-S25. 25th ed. Wayne, Pennysylvani: Clinical and Laboratory Standards Institute; 2015. Performance standards for antimicrobial susceptibility test. Approved Standard.

7. Goel V, Hogade SA, Karadesai SG. Prevalence of extended-spectrum beta-lactamases, AmpC betalactamase, and metallo-beta-lactamase producing Pseudomonas aeruginosa and Acinetobacter baumannii in an intensive care unit in a tertiary care hospital. J Sci Soc 2013;40:28-31.

8. Yong D, Lee K, Yum JH, Shin HB, Rossolini GM, Chong Y, et al. Imipenem-EDTA disk method for differentiation of metallo-beta-lactamase-producing clinical isolates of Pseudomonas spp. and Acinetobacter spp. J Clin Microbiol. 2002;40(!0):3798801.

9. Sabarinathan TV, Rajkumari A. Bacteriological and Resistance Profile In Isolates Of Chronic Suppurative Ottitis Media, Int J Biol Med Res.2015;6(3):5216-8.

10. Mukherjee P, Saunders N, Liu R, Fagan P. Long term outcome of modified radical mastoidectomy. J Laryngol Otol 2004;118:612-6. 
11. Hossian MM, Kundu SC, Haque MR, Shamsuzzaman AK, Khan MK, Halder KK. Extracranial complications of chronic suppurative otitis media. Mymensingh Med J 2006;15(1):4-9.

12. Mansoor T., Musani M., Khalid G., Kamal M. Pseudomonas aeruginosa in Chronic Suppurative Otitis Media: Sensitivity Spectrum against various Antibiotics in Karachi. J Ayub Med Coll Abbottabad 2009;21(2):1203.

13. Amusa YB, Ijaduola IK, Onayade OO. Epidemiology of otitis media in a local tropical African population. West Afr J Med 2005;24(3):227-30.

14. Loy AHC, Tan AL, Lu PKS. Microbiology of chronic suppurative otitis media in Singapore. Singapore Med J 2002;43:296-9.

15. Vineetha Gupta, Abhay Gupta, Sivarajan K. Chronic suppurative otitis media; An aerobic microbiological study. Ind J Otol 1998;4(2):79-82.

16. Nwokoye NN, Egwari L, Olubi CA, Ugoji E, Nwachukwu SC. Predisposing and bacteriological features of otitis media: Afri J Microb Res 2012;6(3):5205.

17. Akinpelu OV, Amusa YB, Komolafe EO, Adeolu AA, Oladele AO, Ameye SA. Challenges in management of Chronic suppurative otitis media in a developing country. J Laryn Otol 2008;122:16-20.

18. Kumar Gulati, Sudesh Kumar, Investigation profile in patients of Chronic suppurative otitis media. Ind J Otol 1997;3(2);59-62.

19. Shahid M, Malik A, Agarwal M, Singhal S. Phenotypic detection of extended spectrum and AmpC betalactamases by a new spot-inoculation method and modified three-dimensional extract test: Comparison with conventional three-dimensional extract test. J Antimicrob Chemother 2004;54:684-7.
20. Syamala R, Sreenivasa Reddy P. The study of bacteriological agents of CSOM: Aerobic culture and evaluation. J Microbiol Biotech Res 2012;2(1):152-62.

21. Singh AH, Basu R, Venkatesh A. Aerobic bacteriology of chronic suppurative otitis media in Rajahmundry, Andhra Pradesh, India. Biol Medi 2012;4(2):73-9.

22. Sanjana RK, Singh YI, Reddy NS. Aerobic bacteriology of chronic suppurative otitis media in a tertiary care hospital: A Retrospective study. J Col Med Sci Nepal 2011;7(2):1-8.

23. Kumar R, Srivastara P, Sharma M, Rishi S, Nirwan PS, Hemwani $\mathrm{K}$, et al. Isolation and Antimicrobial sensitivity profile of bacterial agent in chronic suppurative otitis media patients at NIM Hospital, Jaipur. Int J Pharm Biol Sci 2013;3:265-9.

24. Jeyakumari, D.,Saranya, R., Nagajothi, J. and Selvalingam, E. clinical and bacteriological profile of chronic suppurative otitis media in a rural area of puducherry, India, Int J Devel Res 2015;5(09):5518-22.

25. Mathur P, Tatman A, Das B, Dhavan B. Prevalence of ESBL gram negative bacteria in a tertiary care hospital. Ind J Med Microbiol 2002;115:153-7.

26. Khatoon A, Rizvi M, Sultan A, Khan F, Sharma M, Shukla I, et al. Chronic suppurative otitis media: a clinico-microbiological menace. Int J Res Med Sci 2015;3(8):1932-6. 